\title{
Modelos Volumétricos de Dupla Entrada para Aplicar em Povoamentos Florestais Brasileiros
}

\author{
Valdir Carlos Lima de Andrade ${ }^{1}$ \\ ${ }^{1}$ Curso de Engenharia Florestal, Universidade Federal do Tocantins - UFT, Gurupi/TO, Brasil
}

\begin{abstract}
RESUMO
O objetivo deste trabalho foi apresentar alguns modelos volumétricos de dupla entrada desenvolvidos para quantificar o volume de madeira no Brasil. Como estudo de caso, dados de 180 árvores-amostra de Eucalyptus urophylla e Eucalyptus grandis com idade entre 5 a 7 anos foram utilizados. Para avaliar o desempenho dos modelos volumétricos foram adotados os critérios estatísticos: erro padrão residual, coeficiente de determinação ajustado, média dos erros absolutos, soma de quadrados dos erros relativos, média dos erros percentuais, desvio padrão dos erros, coeficiente de determinação, raiz quadrada do erro médio e análise da distribuição de resíduos. Selecionou-se um modelo volumétrico desenvolvido a partir da equação de fator de forma adaptado ao modelo biomatemático de Gompertz.
\end{abstract}

Palavras-chave: inventário florestal, equações volumétricas, biometria florestal.

\section{Double Entry Volumetric Models for Use in Brazilian Forest Stands}

\begin{abstract}
This study aimed to present some double entry volumetric models developed to quantify the volume of wood in Brazil. As a case study, we used data of 180 trees-sample of Eucalyptus urophylla and Eucalyptus grandis aged 5-7 years. To evaluate the volumetric models performance, we used the following statistical criteria, standard error of the estimate, adjusted coefficient of determination, mean absolute error, sum of squared of relative errors, average of percentage error, standard deviation of the errors, determination coefficient between actual and predicted volume, square root of the average error and analysis of the distribution of residuals. We selected a volumetric model developed from the form factor equation adapted to the Gompertz's biomathematical model.
\end{abstract}

Keywords: forest inventory, volumetric equations, forest biometrics. 


\section{INTRODUÇÃO}

Modelos volumétricos são muito difundidos no Brasil. Estudos sobre eles, incipientes e de grande importância, foram realizados durante a década de 1970, cabendo citar: Veiga (1972), Ferreira et al. (1977), Paula (1977), Siqueira (1977), Higushi et al. (1979), Machado (1979) e Silva \& Schneider (1979). Esses trabalhos, associados ao surgimento dos computadores, alavancaram vários outros na década de 1980, notadamente, méritos devem ser creditados aos trabalhos feitos por: Veiga \& Brasil (1981), Paula et al. (1983), Silva \& Carvalho (1984), Campos et al. (1985) e Couto \& Bastos (1987).

A partir da década de 1990, principalmente, com o avanço tecnológico na área de informática, estudos sobre a aplicabilidade de modelos volumétricos no Brasil intensificaram-se grandemente. Vários desses estudos foram efetuados nas mais variadas situações de povoamentos florestais brasileiros, dentre muitos outros, cabe citar os trabalhos com acácia conduzidos por: Veiga et al. (2000), Schneider \& Tonini (2003) e Muller et al. (2014); trabalhos com eucalipto conduzidos por: Franco et al. (1997), Schneider et al. (1997), Andrade \& Scolforo (2003), Thiersch et al. (2006), Silva et al. (2009), Rocha et al. (2010), Soares et al. (2010), Azevedo et al. (2011a, b) e Miguel et al. (2014); trabalhos com pinus conduzidos por: Silva et al. (1994), Gomes et al. (1997), Couto \& Vettorazzo (1999), Machado et al. (2002), Thomas et al. (2006), Moraes (2009), Pelissari et al. (2011) e Melo et al. (2013); trabalhos com espécies nativas conduzidos por: Scolforo et al. (1994), Rezende et al. (2006), Rolim et al. (2006), Machado et al. (2008), Colpini et al. (2009), Imaña-Encinas et al. (2009), Carvalho et al. (2010), Thaines et al. (2010), Silva et al. (2011), Fraga et al. (2014) e Silva \& Santana (2014).

Diante disso, nota-se que houve uma hegemonia de estudos sobre modelos volumétricos nos últimos 25 anos no Brasil, totalizando $77 \%$ dos trabalhos desenvolvidos. Para aclarar, do total de trabalhos referidos, destacam-se $23 \%$ de estudos desenvolvidos até a década de 1980 (período com cerca de 20 anos), 14\% em cerca de 10 anos (1990 a 1999) e os restantes 63\% nos últimos 15 anos (2000 a 2014). Consequentemente, nesses últimos 45 anos, os modelos volumétricos mais avaliados no Brasil foram: Schumacher e Hall, Stoate,
Spurr, Meyer, Naslund, Ogaya, Takata e Honer. Dentre esses, $41 \%$ dos trabalhos mostraram superioridade do modelo de Schumacher e Hall, 14\%, do modelo Spurr, $17 \%$, do modelo Meyer e 28\%, dos demais. Detalhes desses modelos e respectivas referências bibliográficas podem ser vistos em Veiga (1972), Loetsch et al. (1973) e Miguel et al. (2014).

A situação sobre modelos volumétricos, caracterizada anteriormente, permite inferir sobre um dos motivos pelos quais o modelo volumétrico de Schumacher e Hall é o mais difundido no Brasil. No entanto, como se obtiveram $41 \%$ de resultados favoráveis a esse modelo, então há cerca de $60 \%$ de incertezas no seu emprego como sendo o mais adequado para povoamentos florestais brasileiros. Muitos trabalhos corroboram essa expectativa, conforme se nota nos estudos conduzidos por Veiga (1972), Machado (1979), Silva \& Carvalho (1984), Couto \& Bastos (1987), Scolforo et al. (1994), Veiga et al. (2000), Machado et al. (2002), Andrade \& Scolforo (2003), Schneider \& Tonini (2003), Thiersch et al. (2006), Rezende et al. (2006), Imaña-Encinas et al. (2009), Carvalho et al. (2010), Ferreira et al. (2011), Pelissari et al. (2011), Miguel et al. (2014), dentre muitos outros. Portanto, devido a tais resultados, é preciso cautela ao se utilizar o modelo volumétrico de Schumacher e Hall sem uma prévia comparação com outras opções de modelagem da relação volumétrica.

Diante desse contexto e considerando o significativo avanço da tecnologia de informática, aliado à grande divulgação de conhecimentos técnico-científicos sobre modelos volumétricos e ao fato de a maioria desses terem sido propostos no século passado, entre as décadas de 1930 e 1950, nota-se que é imprescindível desenvolver e/ou criar modelos volumétricos. Assim, o objetivo deste trabalho foi propor novos modelos volumétricos de dupla entrada para aplicação na quantificação do volume de madeira de árvores no Brasil baseando-se nas seguintes hipóteses teóricas:

Hipótese $1\left(\mathrm{HO}_{1}\right)$ : O modelo volumétrico de Schumacher e Hall pode ser utilizado sem prévia comparação com outros modelos volumétricos;

Hipótese $2\left(\mathrm{HO}_{2}\right)$ : É possível desenvolver modelos volumétricos mais eficientes e estáveis que os modelos em realce na literatura florestal brasileira. 


\section{MATERIAL E MÉTODOS}

Visando apresentar uma perspectiva sobre a aplicabilidade dos modelos volumétricos desenvolvidos neste trabalho foi feito um estudo de caso utilizando dados do híbrido entre Eucalyptus urophylla e Eucalyptus grandis (UROG) obtidos na cubagem de 180 árvores-amostra selecionadas em plantios localizados no nordeste da Bahia, com idade entre 5 e 7 anos. Nessas 180 árvores-amostra foram medidos o diâmetro do tronco nas posições: $0,1 \mathrm{~m}, 0,3 \mathrm{~m}, 0,7 \mathrm{~m}, 1,3 \mathrm{~m}$, $2 \mathrm{~m}$ e, a partir daí, a cada metro, até um diâmetro de, aproximadamente, $2 \mathrm{~cm}$.

O cálculo do volume real do fuste das 180 árvores-amostra de UROG foi feito pela aplicação sucessiva da fórmula de Smalian. Já, no cálculo do volume total, acrescentaram-se, ao volume do fuste, o volume do ponteiro e o da cepa, considerados como um cone e um cilindro, respectivamente. Assim, para esses cálculos de volume foram utilizados:

$$
\begin{aligned}
& \left(v_{c p}\right)=\frac{(\pi)\left(d_{0,1}{ }^{2}\right)(0,1)}{(40.000)} \\
& \left(v_{t r}\right)=\sum_{j=1}^{n}\left[\frac{(\pi)\left(d_{1 j}^{2}+d_{2 j}^{2}\right)\left(\ell_{j}\right)}{(80.000)}\right],\left(v_{p t}\right)=\frac{(\pi)\left(d_{n}{ }^{2}\right)\left(\ell_{p t}\right)}{(120.000)}, \\
& \text { e }(v)=v_{c p}+v_{t r}+v_{p t} .
\end{aligned}
$$

em que: $(v c p)=$ volume da cepa, ou, toco; $\pi=$ constante, valendo $3,14159 . . . ; d_{0,1}=$ diâmetro do tronco medido à $0,1 \mathrm{~m}(\mathrm{~cm}) ;(v t r)$ volume do tronco ou fuste, $\mathrm{d}_{1} \mathrm{e}$ $\mathrm{d}_{2}=$ diâmetros medidos nas extremidades de cada j-ésima seção de cubagem $(\mathrm{cm}) ; \ell_{j}=$ comprimento de cada j-ésima seção de cubagem (m); ( $v p t)$ volume do ponteiro ou ponta, $\mathrm{d}_{\mathrm{n}}=$ último diâmetro medido no tronco $(\mathrm{cm}) ; \ell_{p t}=$ comprimento da ponta $(\mathrm{m}) ; \mathrm{e}$ $(v)=$ volume total.

Os volumes totais, obtidos nas 180 árvores-amostra de UROG, foram empregados no ajuste e na avaliação de uma grande gama de modelos volumétricos desenvolvidos e, também, de alguns modelos volumétricos com bom desempenho em povoamentos florestais brasileiros. Para esse propósito, 90 árvores-amostra foram empregadas no ajuste dos modelos volumétricos, outras 90 , com dados independentes, na avaliação da predição do volume. Os modelos volumétricos avaliados, bem como respectivas formas de validação, são descritos a seguir.

\subsection{Proposição de novos modelos para relação volumétrica de árvores}

A base teórica adotada neste trabalho para desenvolver modelos volumétricos foi a quantidade de madeira cabível em um cilindro, obtida a partir da equação de fator de forma. Para isso, inicialmente, a partir dos fatores de forma:

$$
\left(f f_{h}\right)=\frac{(v)}{\left[\frac{\pi(d)^{2}(h)}{40000}\right]} \text { e } \quad f f_{(h-1,3)}=\frac{(v)}{\left[\frac{\pi(d)^{2}(h-1,3)}{40000}\right]},
$$

respectivamente, obtiveram-se:

$$
v 1=\left[\frac{\pi(d)^{2}(h)\left(\overline{f f}_{(h)}\right)}{40000}\right] \text { e } v 2=\left[\frac{\pi(d)^{2}(h-1,3)\left(\overline{f f(}_{(h-1,3)}\right)}{40000}\right] ;
$$

em que $\overline{f f}_{(h)}$ e $\overline{f f}_{(h-1,3)}$ são os fatores de forma médios obtidos para cilindros com comprimento entre $0,0 \mathrm{~m}$ a h e entre 1,3 $\mathrm{m}$ e h, respectivamente, com diâmetro igual ao diâmetro do tronco medido a 1,3 m (d).

Assumindo que a média de v1 e v2 estima melhor o volume, obteve-se que:

$$
v=\frac{\left(\frac{\pi}{40000}\right)\left(d^{2}\right)(h)\left(\overline{f f}_{(h)}\right)+\left(\frac{\pi}{40000}\right)\left(d^{2}\right)(h-1,3)\left(\overline{f f}_{(h-1,3)}\right)}{2} .
$$

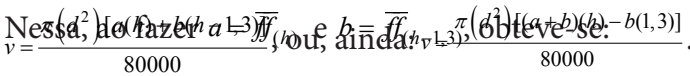

Fazendo-se $b_{0}=\frac{\pi(-b)(1,3)}{80000}$ e $b_{1}=\frac{\pi(a+b)}{80000}$, o resultado é: $\left(v_{i}\right)=\left(d_{i}^{2}\right)\left[b_{0}+b_{1}\left(h_{i}\right)\right]+\left(\varepsilon_{i}\right)$, o qual se trata do modelo volumétrico de Ogaya (1968), cuja em que: i = i-ésima árvore-amostra, $\mathrm{d}=$ diâmetro medido a $1,3 \mathrm{~m}$ do terreno $(\mathrm{cm}), \mathrm{h}=$ altura total $(\mathrm{m}), \mathrm{b}_{0}$ e $\mathrm{b}_{1}=$ coeficientes de regressão a serem estimados e $\varepsilon=$ erro aleatório do modelo utilizado; os demais já foram definidos anteriormente.

O modelo volumétrico de Ogaya, ajustado aos dados de 90 árvores-amostra de UROG, teve suas estimativas estudadas nas diferentes formas funcionais do modelo estatístico: $y_{(i)}=\left(x 1_{i}\right)\left[\delta_{1}+\delta_{2}\left(x 2_{i}\right)\right]+\left(\varepsilon_{i}\right)$. Nesse caso, $y_{(i)}$, ou, $y_{(i)}=\sqrt{v_{i}}$, ou, ainda: $y_{(i)=} v_{(i)} / d_{(i)}$, foi relacionado, com as várias combinações, aos pares das variáveis independentes: $x k_{(i)}, x k_{(i)}=\left(1+d_{i}\right)$, $x k_{(i)}=\operatorname{Ln}\left(d_{i}\right), x k_{(i)}=\left(\frac{1}{d_{i}}\right), x k_{(i)}=\left(h_{i}\right), x k_{(i)}=\left(h_{i}-1\right)$, $x k_{(i)}=\operatorname{Ln}\left(h_{i}\right), x k_{(i)}=\left(\frac{1}{h_{i}}\right), x k_{(i)}=\left(\frac{h_{i}}{d_{i}}\right)$ e $x k_{(i)}=\left(d_{i}^{2} h_{i}\right)$, em que $\mathrm{k}=1$ ou 2 e vice-versa.

Ressalta-se que, esse estudo de modelagem a partir do modelo de Ogaya também foi realizado com a 
aplicação de modelos biomatemáticos nas seguintes formas funcionais:

$y_{i}=x 1_{i}{ }^{\delta_{1}} \delta_{2}\left[1-\exp \left(-\delta_{3} x 2_{i}{ }^{\delta_{4}}\right)\right]^{\delta_{5}}+\varepsilon_{i} \quad$ (1) - Chapman-Richards $y_{i}=\delta_{1}\left[1-\exp \left(-\delta_{2} x_{i}^{\delta_{3}}\right)\right]^{\left[1 /\left(1-\delta_{4}\right)\right]}+\varepsilon_{i}(2)$ - Chapman-Richards

$y_{i}=\delta_{1}\left[1-\exp \left(-\delta_{2} x_{i}^{\delta_{3}}\right)\right]+\delta_{i}$

(3) - Monomolecular

$y_{i}=\frac{\delta_{1}}{1+\exp \left(-\delta_{2} x_{i}^{\delta_{3}}\right)}+\varepsilon_{i}$

(4) - Logística

$y_{i}=\delta_{1}\left[1+\exp \left(-\delta_{2} x_{i}^{\delta_{3}}\right)\right]^{\delta_{4}}+\varepsilon_{i}$

(5) - Logística

$y_{i}=\delta_{1} \exp \left[\exp \left(-\delta_{2} x_{i}^{\delta_{3}}\right)\right]+\varepsilon_{i}$

(6) - Gompertz

$y_{i}=\exp \left\{\frac{\delta_{1}\left[1-\exp \left(-\delta_{2} x_{i}^{\delta_{3}}\right)\right]}{\delta_{4}}\right\}+\varepsilon_{i} \quad$ (7) - Gompertz

em que: $\delta_{1}$ a $\delta_{4}=$ coeficientes a serem estimados, $\mathrm{Ln}=$ logaritmo neperiano, $\exp =$ inverso de Ln e os demais já foram definidos anteriormente.

Ressalta-se, também, que uma grande quantidade de modelos volumétricos foi desenvolvida a partir das várias opções de modelagem geradas com o modelo de Ogaya, com e/ou sem a aplicação dos Modelos 1-7. Assim, numa análise preliminar, a partir da soma de quadrados dos erros e da análise da distribuição de resíduos, foram selecionados 47 novos modelos volumétricos. Desses, com base no coeficiente de determinação ajustado, erro padrão residual e análise da distribuição de resíduos, selecionaram-se os seguintes modelos volumétricos novos:

$$
\begin{aligned}
& (v)=b_{1} d^{\left(\delta_{1}-1\right)} \exp \left[\delta_{2}+(h-1)^{\delta_{3}}\right]+\varepsilon \\
& (v)=(1+d)^{\delta_{1}} \exp \left[\delta_{2}+\delta_{3}\left(\frac{d}{h}\right)\right]+\varepsilon \\
& (v)=\delta_{1}(1+d)^{\delta_{2}} \exp \left[1-\left(\frac{d}{h}\right)^{\delta_{3}}\right]+\varepsilon \\
& (v)=\delta_{1}(1+d)^{\delta_{2}} \exp \left(h^{\delta_{3}}\right)+\varepsilon \\
& \sqrt{(v)}=\left(\frac{\delta_{1}}{\delta_{2}}\right)-\left\{\delta_{1}\left(d+\delta_{3}\right)+\delta_{2}\left[1-\left(d^{2} h\right)^{\delta_{4}}\right]\right\}+\varepsilon \\
& \sqrt{(v)}=\frac{\left[\delta_{1}\left(d^{2} h\right)-\delta_{2}\right]^{\delta_{3}}}{\exp \left[1-\delta_{4}(1 / d)\right]}+\varepsilon \\
& \sqrt{(v)}=\frac{\left[\delta_{1}\left(d^{2} h\right)-\delta_{2}\right]^{\delta_{3}}}{1+\exp \left[-\delta_{4}(1 / d)\right]}+\varepsilon
\end{aligned}
$$

$\sqrt{(v)}=\left[\delta_{1}+(h-1)^{\delta_{2}}\right] \exp \left\{1-\exp \left[\delta_{3}\left(\frac{h}{d}\right)^{\delta_{4}}\right]\right\}+\varepsilon$

$$
\left(\frac{v}{d}\right)=\delta_{1} d^{\left(\delta_{2}-1\right)}\left[1+\exp \left(-\delta_{3} h\right)\right]^{\left(-1 / \delta_{4}\right)}+\varepsilon
$$

$$
\left(\frac{v}{d}\right)=\delta_{1} d^{\left(\delta_{2}-1\right)}\left[\delta_{3}+\left(\delta_{4}-\delta_{3}\right) \exp \left(-\delta_{5} h\right)\right]+\varepsilon
$$

$\left(\frac{v}{d}\right)=d^{\left(\delta_{1}-1\right)}\left[-\delta_{2}+\exp \left(\delta_{3} h\right)\right]+\varepsilon$

$$
\left(\frac{v}{d}\right)=d^{\left(\delta_{1}-1\right)}\left[\delta_{2}+\exp \left(-\delta_{3}+h^{\delta_{4}}\right)\right]+\varepsilon
$$

É importante destacar que os Modelos 8-12, 17-19 foram desenvolvidos a partir do estudo de relações não lineares com as variáveis que contemplam o modelo de Ogaya. Já os Modelos 13-16 caracterizam-se pela aplicação do modelo de Ogaya de forma simultânea com os modelos bio-matemáticos monomolecular e logístico, Modelos 13, 14, Gompertz, no Modelo 15, e de Chapman-Richards, no Modelo 16.

\subsection{Avaliação dos modelos volumétricos}

As equações volumétricas, estimadas a partir dos novos Modelos Volumétricos de 8-19, foram avaliadas por meio de um teste de validação com 90 árvores-amostra, contendo dados diferentes daqueles utilizados no ajuste e seleção preliminar desses modelos. Para esse propósito, foram utilizados os critérios estatísticos: $S Q E R=\sum_{i=1}^{n}\left(\frac{\varepsilon_{i}}{v_{i}}\right)^{2}, M E A=\frac{\sum_{i=1}^{n} \varepsilon_{i}}{n}$, $R^{2}=1-\frac{S Q E}{S Q T}, R Q E M=\sqrt{\left(\frac{S Q E}{n}\right)}$,

$D P E=\sqrt{\left[\sum_{i=1}^{n} \varepsilon_{i}^{2}-\frac{\left(\sum_{i=1}^{n} \varepsilon_{i}\right)^{2}}{n}\right]}$ e $M E P=\left[\sum_{i=1}^{n}\left(\frac{\varepsilon_{i}}{v_{i}}\right) 100\right]\left(\frac{1}{n}\right) ;$ em que $\varepsilon_{i}=\left(v_{i}-\hat{v}_{i}\right), S Q E=\sum_{i=1}^{n} \varepsilon_{i}^{2}, S Q T=\sum_{i=1}^{n} v_{i}^{2}-\frac{\left(\sum_{i=1}^{n} v_{i}\right)^{2}}{n}$, SQER = soma de quadrado dos erros relativos, $\varepsilon i=$ erro ou diferença entre volume real e estimado/predito $\left(\mathrm{m}^{3}\right), v i=$ volume total real $\left(\mathrm{m}^{3}\right), \mathrm{MEA}=$ média dos erros absolutos, $\mathrm{n}=$ número de árvores-amostra utilizadas, $\mathrm{R}^{2}=$ coeficiente de determinação entre volume real e predito, $\mathrm{SQE}=$ soma de quadrados dos erros, $\mathrm{SQT}=$ soma de quadrados total, RQEM = raiz quadrada do erro médio, DPE = desvio padrão dos 
erros, $\mathrm{MEP}=$ média dos erros porcentuais e $v i=$ volume total estimado/predito $\left(\mathrm{m}^{3}\right)$.

Os critérios de avaliação, que foram também utilizados por Andrade \& Leite (2001), Schneider \& Tonini (2003), Imaña-Encinas et al. (2009), Carvalho et al. (2010), Pelissari et al. (2011) e Silva et al. (2011), foram incluídos em um somatório de notas obtidas por classe de diâmetro, que variou conforme a classificação de cada uma das estatísticas analisadas. Nesse caso, o modelo que apresentou a menor soma geral de notas, incluindo-se todas as classes de diâmetro, classificou-se em $1^{\circ}$ lugar.

Visando comparação com os Modelos 8-19, alguns modelos volumétricos em realce na literatura florestal brasileira foram avaliados, destacando-se os seguintes:

$$
\begin{aligned}
& \operatorname{Ln}(v)=b_{0}+b_{1} \operatorname{Ln}(d)+b_{2} \operatorname{Ln}(h)+\operatorname{Ln}(\varepsilon) \\
& (v)=b_{0}+b_{1}\left(d^{2} h\right)+(\varepsilon) \\
& (v)=b_{0}+b_{1}(d)+b_{2}\left(d^{2}\right)+b_{3}(d h)+b_{4}\left(d^{2} h\right)+(\varepsilon) \\
& (v)=b_{0}+b_{1}\left(d^{2}\right)+b_{2} \operatorname{Ln}\left(d^{2} h\right)+b_{3}\left(d h^{2}\right)+b_{4}\left(h^{2}\right)+(\varepsilon) \\
& (v)=\frac{\left(d^{2} h\right)}{\left[b_{0}+b_{1}(d)\right]}+(\varepsilon) \\
& (v)=(d)^{2}\left[b_{0}+b_{1}(h)\right]+(\varepsilon)
\end{aligned}
$$

Os Modelos Volumétricos 20-25, que se referem aos modelos de Schumacher e Hall, Spurr, Meyer, Näslund, Takata e Ogaya, respectivamente, foram considerados usuais para efeito de comparação porque, conforme explanado na introdução deste estudo, representam cerca de $75 \%$ dos trabalhos que apresentaram tais modelos como os mais adequados para quantificar o volume de madeira no Brasil.

Outros dois novos modelos, gerados a partir de 9 e 11, foram incluídos nessa avaliação: o Modelo 26 é uma forma linear do Modelo 9 e o Modelo 27, uma forma linear aproximada do Modelo 11:

$$
\begin{aligned}
& \operatorname{Ln}(v)=b_{0}+b_{1}\left(\frac{d}{h}\right)+b_{2} \operatorname{Ln}(1+d)+\operatorname{Ln}(\varepsilon) \\
& \operatorname{Ln}(v)=b_{0}+b_{1} \operatorname{Ln}(h)+b_{2} \operatorname{Ln}(1+d)+\operatorname{Ln}(\varepsilon)
\end{aligned}
$$

Destaca-se que, devido às transformações feitas na variável dependente dos Modelos 12-20, 26, 27, o erro padrão residual (EPR) e o coeficiente de determinação ajustado $\left(\mathrm{R}^{2} \mathrm{aj}\right)$ foram recalculados, aplicando-se: $E P R=\sqrt{\left(\frac{S Q E}{n-p}\right)}$ e $R^{2} a j=1-\left(\frac{S Q E}{S Q T}\right)\left[\frac{(n-1)}{(n-p)}\right]$, em que: $\mathrm{b}_{0} \mathrm{a}_{4}=$ parâmetros a serem estimados, $\mathrm{p}=$ número de coeficientes a serem estimados no modelo; os demais já foram definidos anteriormente.

\section{RESULTADOS E DISCUSSÃO}

Os dados obtidos, divididos em seis classes de diâmetro com amplitude de $3 \mathrm{~cm}$ e tendo 30 árvores-amostra cada, representaram uma variação de diâmetros entre 5,1 e $22,9 \mathrm{~cm}$ e de alturas totais entre 10,3 e $32,0 \mathrm{~m}$ (Tabela 1).

Os resultados obtidos para os Modelos 8-19 (Tabela 2) mostram que houve um bom ajuste desses novos modelos, para os quais, numa análise preliminar

\begin{tabular}{|c|c|c|c|c|c|c|c|c|c|}
\hline \multirow{2}{*}{$\begin{array}{c}\mathrm{cd} \\
(\mathrm{cm})\end{array}$} & \multicolumn{8}{|c|}{ Classe de altura (m) } & \multirow{2}{*}{ Total } \\
\hline & 9,5 & 12,5 & 15,5 & 18,5 & 21,5 & 24,5 & 27,5 & 30,5 & \\
\hline 6,5 & $5(6)$ & $9(6)$ & $1(3)$ & - & - & - & - & - & $15(15)$ \\
\hline 9,5 & $0(2)$ & $6(6)$ & $5(4)$ & $4(3)$ & - & - & - & - & $15(15)$ \\
\hline 12,5 & - & $3(2)$ & $3(7)$ & $5(3)$ & $3(2)$ & $1(1)$ & - & - & $15(15)$ \\
\hline 15,5 & - & - & $2(3)$ & $4(4)$ & $8(5)$ & $0(2)$ & $1(1)$ & - & $15(15)$ \\
\hline 18,5 & - & - & $1(0)$ & $3(1)$ & $3(4)$ & $4(9)$ & $4(1)$ & - & $15(15)$ \\
\hline 21,5 & - & - & - & $0(2)$ & $2(4)$ & $7(7)$ & $2(2)$ & $4(-)$ & $15(15)$ \\
\hline Total & $5(8)$ & $18(14)$ & $12(17)$ & $16(13)$ & $16(15)$ & $12(19)$ & $7(4)$ & $4(0)$ & $90(90)$ \\
\hline
\end{tabular}
associada à distribuição de resíduos, aceitou-se $E P R \leq 8 \%$ e Rªj $\geq 99 \%$. Nessa Tabela 2, também, estão apresentados os resultados dos três melhores modelos

Tabela 1. Distribuição de frequência de 180 árvores-amostra de UROG separadas em lotes de dados de ajuste e validação.

Table 1. Frequency distribution of 180 sample trees of UROG separated in the data set for adjustment and for validation.

cd = classe de diâmetro; valores entre parênteses $=$ dados de validação. 
Tabela 2. Estatísticas obtidas para 17 modelos volumétricos, sendo 14 modelos novos e 3 considerados usuais em análises volumétricas do tronco de árvores no Brasil.

Table 2. Statistics obtained for 17 volumetric models, 14 new models and 3 considered usual in volumetric analysis of the trunk of trees in Brazil.

\begin{tabular}{|c|c|c|c|c|c|c|c|c|c|}
\hline \multicolumn{10}{|c|}{ Novos modelos volumétricos não lineares } \\
\hline md & $\hat{\delta}_{1}$ & $\hat{\delta}_{2}$ & $\hat{\delta}_{3}$ & $\hat{\delta}_{4}$ & $\hat{\delta}_{5}$ & SQE & EPR & $\mathbf{R}^{2} \mathbf{a j}$ & NG \\
\hline 8 & 2,776 & $-10,519$ & 0,357 & - & - & 0,01633 & 7,94 & 99,05 & $250^{3}$ \\
\hline 9 & $-9,190$ & $-1,424$ & 3,045 & - & - & 0,01617 & 7,90 & 99,06 & 333 \\
\hline 10 & 1,596 & $2,60 \mathrm{E}-05$ & 3,024 & - & - & 0,01637 & 7,95 & 99,04 & 388 \\
\hline 11 & 0,361 & $4,4 \mathrm{E}-05$ & 1,893 & - & - & 0,01625 & 7,92 & 99,05 & 364 \\
\hline 12 & 0,0056 & 98,592 & 0,00977 & 0,462 & - & 0,01740 & 8,24 & 98,97 & $230^{2}$ \\
\hline 13 & 0,0181 & $1,86 \mathrm{E}-04$ & 0,526 & 2,904 & - & 0,01709 & 8,17 & 98,99 & $292^{5}$ \\
\hline 14 & $4,10 \mathrm{E}-04$ & $8,00 \mathrm{E}-05$ & 0,559 & 13,740 & - & 0,01677 & 8,09 & 99,01 & $250^{3}$ \\
\hline 15 & 5,530 & 1,451 & 1,793 & 0,0771 & - & 0,01667 & 8,07 & 99,02 & $187^{1}$ \\
\hline 16 & 0,00567 & 1,785 & 0,0423 & 0,240 & - & 0,01669 & 8,07 & 99,01 & 350 \\
\hline 17 & 0,0123 & 1,777 & $-0,229$ & 0,011 & $-0,0168$ & 0,01646 & 8,06 & 99,02 & 306 \\
\hline 18 & 1,774 & 1,0001 & $7,2 \mathrm{E}-05$ & - & - & 0,01790 & 8,31 & 98,96 & 312 \\
\hline 19 & 1,776 & $-1,1 \mathrm{E}-04$ & 9,369 & 0,346 & - & 0,01652 & 7,98 & 99,04 & 296 \\
\hline \multicolumn{10}{|c|}{ Modelos volumétricos usuais } \\
\hline md & $\hat{b}_{0}$ & $\hat{b}_{1}$ & $\hat{b}_{2}$ & $\hat{b}_{3}$ & $\hat{b}_{4}$ & SQE & EPR & $\mathbf{R}^{2} \mathbf{a j}$ & NG \\
\hline 20 & $-9,779$ & 1,766 & 1,062 & - & - & 0,01803 & 8,34 & 98,95 & $274^{4}$ \\
\hline 23 & $-1,74 \mathrm{E}-02$ & $2,48 \mathrm{E}-04$ & $5,77 \mathrm{E}-03$ & $2,88 \mathrm{E}-05$ & $-2,45 \mathrm{E}-04$ & 0,01772 & 8,27 & 98,97 & 484 \\
\hline 25 & $1,41 \mathrm{E}-04$ & $2,93 \mathrm{E}-05$ & - & - & - & 0,02277 & 9,37 & 98,67 & 489 \\
\hline \multicolumn{10}{|c|}{ Novos modelos volumétricos lineares } \\
\hline md & $\hat{b}_{0}$ & $\hat{b}_{1}$ & $\hat{b}_{2}$ & - & - & SQE & EPR & $\mathbf{R}^{2} \mathbf{a j}$ & NG \\
\hline 26 & $-9,2056$ & $-1,3369$ & 3,0285 & - & - & 0,01646 & 7,97 & 99,04 & 317 \\
\hline 27 & $-10,3215$ & 1,0527 & 1,9281 & - & - & 0,01730 & 8,17 & 98,99 & 297 \\
\hline
\end{tabular}

$\mathrm{md}=\mathrm{n}$ do modelo; $\mathrm{NG}=$ nota geral obtida incluindo-se todas as classes de diâmetro, cujos números sobrescritos 1 a 5 referem-se à classificação do modelo de acordo com o menor resultado de NG.

usuais, quais sejam: modelos de Schumacher e Hall (20), Näslund (23) e Ogaya (25). Tais modelos, apesar de $\mathrm{R}^{2}$ aj e EPR estarem fora dos limites preestabelecidos, apresentaram boa distribuição de resíduos, similar às situações obtidas com os Modelos 12-18.

Na Tabela 2, também, observa-se que NG $=187$ indica o Modelo 15 como o melhor de todos, seguido dos Modelos 12, 8, 14, classificados em segundo e terceiros lugares, com 230, 250 e 250 pontos, respectivamente. Dentre os modelos usuais, o Modelo 20 foi o que melhor se classificou, ficando em quarto lugar, com NG de 274 pontos.

Os resultados obtidos para os modelos selecionados 15 e 20 (Tabela 2) foram iguais para $R^{2} a j$, sendo de 0,990 e EPR de 8,07\% e 8,34\%, respectivamente. Em trabalhos, exclusivamente com eucalipto, conforme se nota em: Franco et al. (1997), Schneider et al. (1997), Andrade \& Leite (2001), Leite \& Andrade (2003), Thiersch et al.
(2006), Rocha et al. (2010), Azevedo et al. (2011a), Miguel et al. (2014) e Muller et al. (2014), as estatísticas $\mathrm{R}^{2}$ aj e EPR variaram de 0,875 a 0,994 e de $4,76 \%$ a $10,37 \%$. Portanto, valores que englobam os resultados obtidos neste estudo.

Especificamente, para eucalipto com idade dentro das que compõem este estudo, conforme se nota em: Schneider et al. (1997), Andrade \& Leite (2001), Leite \& Andrade (2003), Thiersch et al. (2006), Soares et al. (2010), Rocha et al. (2010), Azevedo et al. (2011b) e Miguel et al. (2014), os valores de Rªj e EPR variaram entre 0,910 a 0,995 e de $4,66 \%$ a $9,16 \%$, respectivamente. Portanto, para os Modelos Volumétricos 15, 20, os valores de Rªj e EPR (Tabela 2) estão dentro do nível de precisão nos seus ajustes.

A análise da distribuição de resíduos, de maneira geral, mostrou para o Modelo 15 um comportamento mais homogêneo ao longo do eixo zero, tendo a grande 
maioria dos resíduos oscilado em $\pm 10 \%$ (Figura 1a versus Figura 1c), com proximidade de $95 \%$ para o Modelo 15 e de $81 \%$ para o Modelo 20 (Figura $1 \mathrm{~b}$ versus Figura 1d). No caso de se considerarem erros oscilando em $\pm 5 \%$, essa situação apresenta proximidade de $65 \%$ para o modelo 15 e de $48 \%$ para o Modelo 20 (Figura $1 \mathrm{~b}$ versus Figura 1d). Essa análise da Figura 1, complementando a da Tabela 2, indica o Modelo 15 como o melhor dentre todos os modelos volumétricos avaliados neste trabalho.

A Figura 2 indica que o Modelo 15 apresenta a menor variação dos erros em torno do eixo zero, com tendência à subestimação de forma menos pronunciada que o Modelo 20. Assim, entre as avaliações de um grande número de equações, as estimativas obtidas para o Modelo 15 revelaram-se como as de melhor desempenho, ou seja:

$$
\hat{v}=\left\{\left[5,5295+(h-1)^{1,4510}\right] \exp \left\{1-\exp \left[1,7931\left(\frac{h}{d}\right)^{0,0771}\right]\right)\right\}^{2}
$$

Na comparação entre os Modelos 10, 11, que têm a mesma base $\delta_{1}(1+d)^{\delta_{3}}$, nota-se superioridade do Modelo 11, com 364 pontos contra 388 do Modelo 10. Análise similar também pode ser feita com os Modelos 13, 14, os quais têm o mesmo numerador: $\delta_{1}(1+d)^{\delta_{2}}$. Nota-se que o Modelo 14 é o melhor, com 250 pontos contra 292 do Modelo 13. Também pode-se fazer a comparação entre os Modelos 16, 17, os quais têm a mesma base: $\delta_{1} d^{\left(\delta_{2}-1\right)}$. Nota-se que o Modelo 17 é melhor, com 306 pontos contra 350 do modelo 16. Por fim, na comparação entre os Modelos 18 e 19, os quais têm a mesma base: $d^{\left(\delta_{1}-1\right)}$, nota-se que o Modelo 19 é melhor, com 296 pontos contra 312 do Modelo 18.

Confrontando-se os dois novos Modelos 26, 27 com os Modelos 9, 11, nota-se que houve redução de NG de 333 pontos (Modelo 9) para 317 (Modelo 26) e de 364 pontos (Modelo 11) para 297 (Modelo 27). Isso indica que se deve preferir o ajuste linear e que, entre os Modelos 26, 27, a variável $\operatorname{Ln}(\mathrm{h}$ ) é preferível à variável $\left(\frac{d}{h}\right)$ devido o Modelo 27 apresentar o menor NG (297 contra 317) e melhor distribuição de resíduos, pois tais modelos diferem apenas na variável independente que acompanha o parâmetro $b_{1}$.

Diante das análises feitas neste estudo, pôde-se decidir pela rejeição da hipótese $\mathrm{HO}_{1}$ e aceitação da hipótese $\mathrm{HO}_{2}$ ressaltando-se que, se fossem avaliados somente modelos volumétricos em realce na literatura brasileira, o modelo de Schumacher e Hall seria selecionado, implicando na aceitação da hipótese $\mathrm{HO}_{1}$. No entanto, como alguns dos novos modelos volumétricos foram superiores ao de Schumacher e Hall, resultando na aceitação da hipótese $\mathrm{HO}_{2}$, então sempre se devem comparar vários modelos de volumes, independentemente do objetivo a que se refere o uso de modelos volumétricos no Brasil.

É claro que, em futuras repetições deste estudo, baseadas em outras espécies florestais e/ou diferentes situações de povoamento florestal, poderão ocorrer decisões diferentes. Por isso, em outros estudos de caso

\section{a)-Modelo 15}

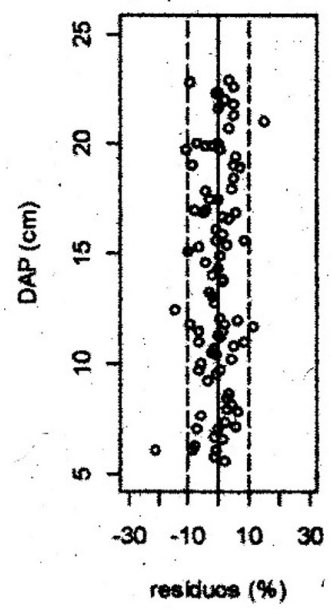

b) - Modelo 15

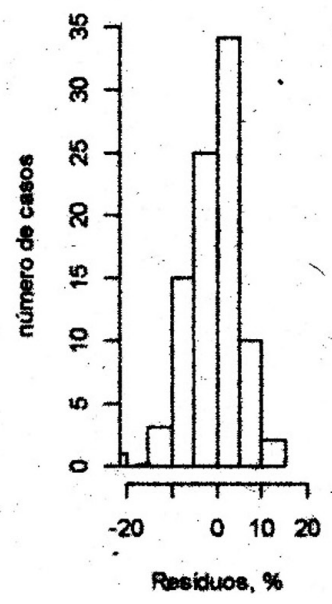

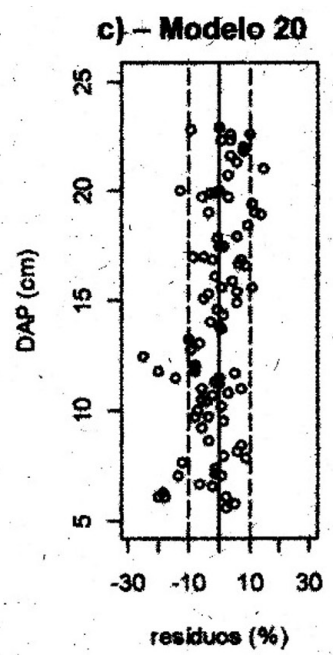

\section{d) - Modelo 20}

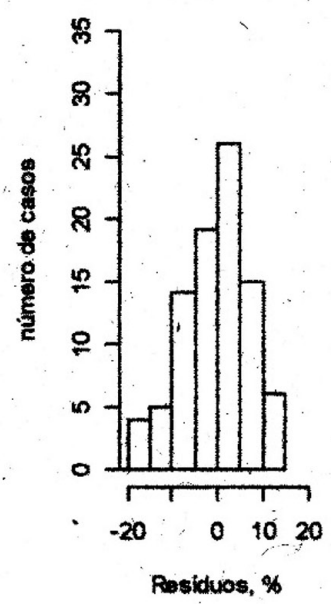

Figura 1. Distribuição de resíduos para os Modelos 15, 20. Figure 1. Distribution of residuals for Models 15, 20. 


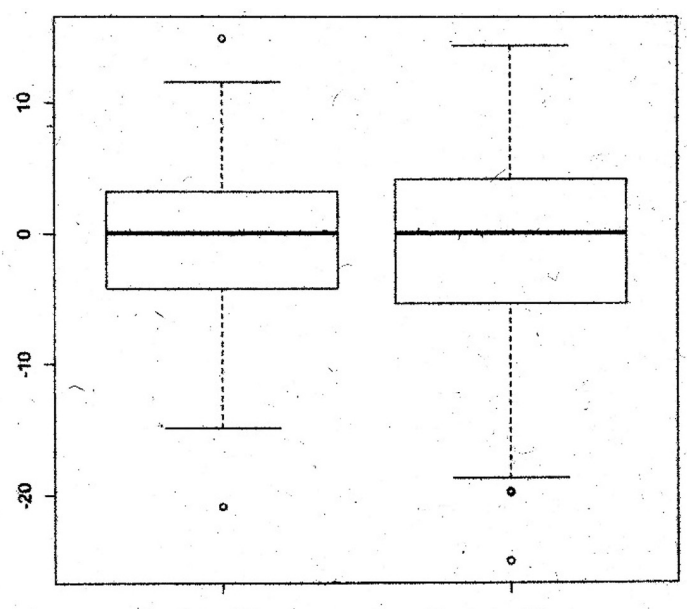

- Modelo 15

Figura 2. Esquema dos cinco números obtidos para os Modelos 15, 20 (valores residuais em porcentagem: mínimo, primeiro quartil, mediana, terceiro quartil e máximo).

Figure 2. Layout of the five numbers obtained for Models 15, 20 (residual values in percent: minimum, first quartile, median, third quartile and maximum).

com avaliação de modelos volumétricos sugere-se que sejam novamente avaliadas as hipóteses $\mathrm{H}_{1}$ e $\mathrm{HO}_{2}$.

\section{CONCLUSÕES}

Os resultados obtidos permitiram concluir que o Modelo 15, de formulação $\sqrt{(v)}=\left[\delta_{1}+(h-1)^{\delta_{2}}\right] \exp \left\{1-\exp \left[\delta_{3}\left(\frac{h}{d}\right)^{\delta_{4}}\right]\right\}+\varepsilon$, é o mais adequado para o clone UROGR em condições semelhantes a este estudo de caso. Isso levou à rejeição da hipótese $\mathrm{H}_{1}$ e à aceitação da hipótese $\mathrm{H}_{2}$. Também pôde-se concluir que:

- Dentre os modelos em realce no Brasil, o modelo de Schumacher e Hall foi o de melhor desempenho;

- Dentre todos os modelos volumétricos avaliados, o modelo de Schumacher e Hall foi classificodo em $4^{\circ}$ lugar;

- Os Modelos Volumétricos 8, 12 e 14, das formulações $(v)=b_{1} d^{\left(\delta_{1}-1\right)} \exp \left[\delta_{2}+(h-1)^{\delta_{3}}\right]+\varepsilon, \mathrm{e} \sqrt{(v)}=\frac{\left[\delta_{1}\left(d^{2} h\right)-\delta_{2}\right]^{\delta_{3}}}{1+\exp \left[-\delta_{4}(1 / d)\right]}+\varepsilon$, respectivamente, além do Modelo 15, foram superiores ao modelo de Schumacher e Hall. Portanto, o modelo de Schumacher \& Hall só pode ser utilizado, sem precisar de comparação com outros modelos volumétricos, se tais modelos já são existentes antes dos modelos desenvolvidos neste trabalho e, ou, em outros que podem surgir;

- É sempre necessário avaliar vários modelos volumétricos a fim de se decidir pelo mais apropriado ao cenário de estudo;

- Em análise de modelos volumétricos não se pode deixar de fazer a análise da distribuição de resíduos atrelada às estatísticas de precisão e/ou de validação de equações volumétricas ajustadas.

\section{STATUS DA SUBMISSÃO}

Recebido: 2 mar., 2015

Aceito: 23 maio, 2016

\section{AUTOR(ES) PARA CORRESPONDÊNCIA}

\section{Valdir Carlos Lima de Andrade}

Curso de Engenharia Florestal, Universidade

Federal do Tocantins - UFT, Rua Badejos, 17, chác. 69/72, Zona Rural, CP 66, CEP 77402-970, Gurupi, TO, Brasil

e-mail: vclandrade@hotmail.com

\section{REFERÊNCIAS}

Andrade VCL, Leite HG. Uso da geometria analítica para quantificação do volume de árvores individuais. Revista Árvore 2001; 24(4): 481-486.

Andrade VCL, Scolforo JRS. Identidade e acurácia de equações volumétricas obtidas por meio de geometria analítica. In: Anais da $48^{a}$ RBRAS - Reunião Anual da Região Brasileira da Sociedade Internacional de Biometria e 10 SEAGRO - Simpósio de Estatística Aplicada à Experimentação Agronômica [CD-ROM]; 2003; Lavras. Lavras: Universidade Federal de Lavras; 2003.

Azevedo GB, Souza GTO, Barreto PAB, Conceição V Jr. Estimativas volumétricas em povoamentos de Eucalipto sob regime de alto fuste e talhadia no sudoeste da Bahia. Pesquisa Florestal Brasileira 2011b; 31(68): 309-318. http:// dx.doi.org/10.4336/2011.pfb.31.68.309.

Azevedo TL, Mello AA, Ferreira RA, Sanquetta CR, Nakajima NY. Equações hipsométricas e volumétricas para um povoamento de Eucalyptus sp. localizado na FLONA do Ibura, Sergipe. Revista Brasileira de Ciências Agrárias 2011a; 6(1): 105-112. http://dx.doi.org/10.5039/ agraria.v6i1a861. 
Campos JCC, Trevizol TL Jr, Paula F No. Ainda, sobre seleções de equações de volume. Revista Árvore 1985; 9: 115-126.

Carvalho SPC, Mendonça AR, Lima MP, Calegario N. Differente strategies to estimate the commercial volume of Anadenanthera colubrina (Vell.) Brenan. Cerne 2010; 16(3): 399-406. http://dx.doi.org/10.1590/S010477602010000300016 .

Colpini C, Travagin DP, Soares TS, Silva VSM. Determinação do volume, do fator de forma e da porcentagem de casca de árvores individuais em uma Floresta Ombrófila Aberta na região noroeste de Mato Grosso. Acta Amazônica 2009; 39(1): 97-104.

Couto HTZ, Bastos NLM. Modelos de equações de volume e relações hipsométricas para plantações de Eucalyptus no estado de São Paulo. IPEF 1987; 37: 33-44.

Couto HTZ, Vettorazzo SC. Seleção de equações de volume e peso seco comercial para Pinus taeda. Cerne 1999; 5: 69-80.

Ferreira CA, Mello HA, Kajiya S. Estimativa do volume de madeira aproveitável para celulose em povoamentos de Eucalyptus spp.: determinação de equações para o cálculo do volume de povoamentos de Eucalyptus spp. IPEF 1977; 14: 29-50.

Ferreira JCS, Silva JAA, Miguel EP, Imaña Encinas J, Tavares JA. Eficiência relativa de modelos volumétricos com e sem a variável altura das árvores. Revista Acta Tecnológica 2011; 6(1): 89-102.

Fraga MP, Barreto PAB, Paula A. Estimação de volume de pterogyne nitens em plantio puro no sudoeste da Bahia. Pesquisa Florestal Brasileira 2014; 34(79): 207-215. http:// dx.doi.org/10.4336/2014.pfb.34.79.703.

Franco EJ, Scolforo JRS, Mello JM, Oliveira AD. Eficiência dos métodos para estimativa volumétrica de Eucalyptus camaldulensis. Cerne 1997; 3(1): 82-117.

Gomes FS, Maestri R, Sanqueta CR. Avaliação da produção em volume total e sortimento em povoamentos de Pinus taeda L. submetidos a diferentes condições de espaçamento inicial e sítio. Ciência Florestal 1997; 7(1): 101-126.

Higushi N, Gomes B, Santos J, Constantino NA. Tabela de volume para povoamento de Eucalyptus grandis plantado no município de Várzea Grande, (MT). Floresta 1979; 10(1): 43-44.

Imaña-Encinas J, Santana AO, Paula JE, Imaña CR. Equações de volume de madeira para o cerrado de Planaltina de Goiás. Floresta 2009; 39(1): 107-116. http:// dx.doi.org/10.5380/rf.v39i1.13731.

Leite HG, Andrade VCL. Importância das variáveis altura dominante e altura total em equações hipsométricas e volumétricas. Revista Árvore 2003; 27(3): 301-310. http:// dx.doi.org/10.1590/S0100-67622003000300005.

Loetsch F, Zohrer F, Haller KE. Forest inventory. 2nd ed. Manchem: BLV; 1973. 479 p.
Machado SA, Conceição MB, Figueiredo DJ. Modelagem do volume individual para diferentes idades e regimes de desbaste em plantações de Pinus oocarpa. Ciências Exatas e Naturais 2002; 4(2): 185-196.

Machado SA, Figura MA, Silva LCR, Teo SL, Stolle L, Urbano E. Modelagem volumétrica para bracatinga (Mimosa scabrella) em povoamentos da região metropolitana de Curitiba. Pesquisa Florestal Brasileira 2008; 56: 17-29.

Machado SA. Estimativa de sobrevivência de Pinus taeda em plantios homogêneos. Floresta 1979; 10(1): 73-76.

Melo LC, Barreto PAB, Oliveira FGRB, Novaes AB. Estimativas volumétricas em povoamento de Pinus caribaea var. hondurensis no sudoeste da Bahia. Pesquisa Florestal Brasileira 2013; 33(76): 379-386. http://dx.doi. org/10.4336/2013.pfb.33.76.459.

Miguel EP, Lea FA, Hono HA, Leal UAS. Modelagem na predição do volume individual em plantio de Eucalyptus urograndis. Revista Brasileira de Biometria 2014; 32(4): 584-598.

Moraes SP No. Estimativas de volume de Pinus tecunumanii no Cerrado do Distrito Federal. Planaltina: Embrapa Cerrados; 2009. 16 p. Boletim de Pesquisa e Desenvolvimento, 231.

Muller MD, Salles TT, Paciulo DSC, Brighenti AM, Castro CRT. Equações de altura, volume e afilamento para eucalipto e acácia estabelecidos em sistema silvipastoril. Floresta 2014; 44(3): 473-484. http://dx.doi.org/10.5380/ rf.v44i3.33149.

Ogaya N. Kubierungs formeln and best andesmassen formeln [thesis]. Freiburg: Forest Science, University Freiburg; 1968.

Paula F No, Souza AL, Quintaes PCG, Soares VP. Análise de equações volumétricas para Eucalyptus spp, segundo o método de regeneração na região de José de Melo-MG. Revista Árvore 1983; 7(1): 56-70.

Paula F No. Tabelas volumétricas com e sem casca para Eucalyptus saligna. Revista Árvore 1977; 1(1): 31-54.

Pelissari AL, Lanssanova LR, Drescher R. Modelos volumétricos para Pinus tropicais, em povoamento homogêneo, no Estado de Rondônia. Pesquisa Florestal Brasileira 2011; 31(67): 173-181. http://dx.doi.org/10.4336/2011.pfb.31.67.173.

Rezende AV, Vale AT, Sanquetta CR, Figueiredo A Fo, Felfili JM. Comparação de modelos matemáticos para estimativa do volume, biomassa e estoque de carbono da vegetação lenhosa de um cerrado sensu stricto em Brasília, DF. Scientia Forestalis 2006; 71: 65-76.

Rocha TB, Cabacinha CD, Almeida RC, Paula A, Santos RC. Avaliação de métodos de estimativa de volume para um povoamento de Eucalyptus urophylla s. T. Blake no Planalto da Conquista-BA. Enciclopédia Biosfera 2010; 6: 1-13.

Rolim SG, Couto HTZ, Jesus RM, Franca JT. Modelos volumétricos para a floresta nacional do Tapirapé-aquirí, 
Serra dos Carajás (PA). Acta Amazonica 2006; 36(1): 107 114. http://dx.doi.org/10.1590/S0044-59672006000100013.

Schneider PR, Coelho MCB, Zanon MLB, Finger CAG, Klein JEM. Equações de volume para Eucalyptus dunnii Maiden, determinadas para a Depressão Central do Estado do Rio Grande do Sul. Ciência Rural 1997; 27(3): 425-428. http://dx.doi.org/10.1590/S0103-84781997000300010.

Schneider PR, Tonini H. Utilização de variáveis dummy em equações de volume para Acacia mearnsii De Wild. Ciência Florestal 2003; 13(2): 121-129.

Scolforo JRS, Mello JM, Lima CSA. Obtenção de relações quantitativas para estimativa de volume do fuste em floresta estacional semidecídua montana. Cerne 1994; 1(1): 123-134.

Silva EM, Santana AC. Modelos de regressão para estimação do volume de árvores comerciais, em florestas de Paragominas. Revista Ceres 2014; 61(5): 631-636. http:// dx.doi.org/10.1590/0034-737X201461050005.

Silva ENS, Santana AC, Queiroz WT, Souza RJ. Estimação de equações volumétricas para árvores de valor comercial em Paragominas, estado do Pará. Amazônia. Ciência \& Desenvolvimento 2011; 7(13): 7-18.

Silva JAA, Machado SA, Borders BE. Aumento da precisão de modelos volumétricos através do uso da transformação de Box e Cox. Cerne 1994; 1(1): 13-16.

Silva JNM, Carvalho MSP. Equações de volume para uma floresta secundária no planalto do Tapajós-Belterra, PA. Boletim de Pesquisa Florestal 1984; 8/9: 1-15.

Silva JNM, Schneider PR. Comparação de equações de volume para povoamento de Acacia mearnsii de Wild (Acácia negra) no Estado do Rio Grande do Sul. Floresta 1979; 10(1): 36-42.
Silva MLM, Binoti DHB, Gleriani JM, Leite HG. Ajuste do modelo de Schumacher e Hall e aplicação de redes neurais artificiais para estimar volume de árvores de eucalipto. Revista Árvore 2009; 33(6): 1133-1139. http:// dx.doi.org/10.1590/S0100-67622009000600015.

Siqueira JDP. Tabelas de volume para povoamentos nativos de Araucaria angustifolia (Bert) O. Ktze, no sul do Brasil. Floresta 1977; 8(1): 7-12.

Soares CPB, Silva GF, Martins FB. Influence of section lengths on volume determination in Eucalyptus trees. Cerne. Lavras 2010; 16(2): 155-162.

Thaines F, Braz EM, Mattos PV, Thaines AAR. Equações para estimativa de volume de madeira para a região do Rio Ituxi, Lábrea, AM. Pesquisa Florestal Brasileira 2010; 30(64): 283-289. http://dx.doi.org/10.4336/2010.pfb.30.64.283.

Thiersch CR, Scolforo JRS, Oliveira AD, Maestri R, Rezende GDSP. Acurácia dos métodos para estimativa do volume comercial de clones de Eucalyptus sp. Cerne 2006; 12: 167-181.

Thomas C, Andrade CM, Schneider PR, Finger CAG. Comparação de equações volumétricas ajustadas com dados de cubagem e análise de tronco. Ciência Florestal 2006; 16(3): 319-327. http://dx.doi.org/10.5902/198050981911.

Veiga RAA, Brasil MAM. Peso de matéria seca e volume do Eucalyptus propinqua Deane ex Maiden: equações. Boletim Técnico: Instituto Florestal 1981; 35(2): 73-84.

Veiga RAA, Carvalho CM, Brasil MAM. Determinação de equações de volume para árvores de Acacia mangium Wild. Cerne 2000; 6: 103-107.

Veiga RAA. Comparação de equações de volume para Eucalyptus saligna Smith. I: equações aritméticas não formais. Floresta 1972; 4(1): 81-94. 